\title{
SYNTHETIC TEMPLATE SPECTRA
}

\author{
ROBERT L. KURUCZ \\ Harvard-Smithsonian Center for Astrophysics \\ 60 Garden Street, Cambridge, MA 02138, U.S.A.
}

I will compute representative spectra for all spectral types for which my model calculations (Kurucz 1992; 1993a; 1994a) are valid. High-resolution zero-rotation fluxes and intensities will be distributed on CD-ROMS together with programs (Kurucz 1993B) for broadening the spectra to arbitrary rotation velocities, macroturbulent velocities, and instrumental profiles, and for transmitting the spectrum through an atmosphere.

Since the line data are still not well determined, I actually have to make two calculations, one with only the lines with good wavelengths to preserve wavelength detail, and one with all lines, including predicted, to preserve the energy distribution for photometric calibration. In the first case the spectrum is usable as a radial velocity, abundance, or classification template. Even though the line list is incomplete, its signal-to-noise can be higher than that of observed spectra for cross-correlating.

The models are plane parallel, LTE, in hydrostatic equilibrium, and have no triatomic opacities. Therefore, $M$ stars and supergiants cannot be computed realisticly. The abundances available now are scaled-solar and alpha-enhanced scaled-solar. Individual abundances can be changed for the spectrum calculation. My new model atmosphere program, ATLAS12 (Kurucz 1993c), which does not yet work, will allow arbitrary abundances.

I have made test calculations for Arcturus, the sun, Vega, Sirius, and a 17000K B star. Figure 1 shows the effect of resolution on the appearance of the spectrum. Figure 2 shows the effect of rotation. The spectrum for Arcturus is displayed in Kurucz (1994b) with the molecular bands labelled.

\section{References}

Kurucz, R.L. (1992) Model atmospheres for population synthesis. in Stellar Population of Galaxies, eds. B. Barbuy and A. Rensini, Kluwer, Dordrecht, pp. 225-232.

Kurucz, R.L. (1993a) ATLAS9 Stellar Atmosphere Programs and $2 \mathrm{~km} / \mathrm{s}$ grid, Kurucz CD-ROM No.13.

Kurucz, R.L. (1993b) SYNTHE Spectrum Synthesis Programs and Line Data, Kurucz CD-ROM No.18.

Kurucz, R.L. (1993c) A new opacity-sampling model atmosphere program for arbitrary abundances. in Peculiar versus Normal Phenomena in A-type and Related Stars, eds. M.M. Dworetsky, F. Castelli, and R. Faraggiana, A.S.P. Conference Series vol. 44, San Francisco, Astronomical Society of the Pacific, pp. 87-97.

Kurucz, R.L. (1994a) Solar abundance model atmospheres for $0,1,2,4,8 \mathrm{~km} / \mathrm{s}$, Kurucz CD-ROM No. 19.

Kurucz, R.L. (1994b) Computation of opacities for diatomic molecules, in IAU Colloquium 146: Molecules in the Stellar Environment, ed. U.G. Jorgensen. SpringerVerlag, Berlin, pp. 282-295. 


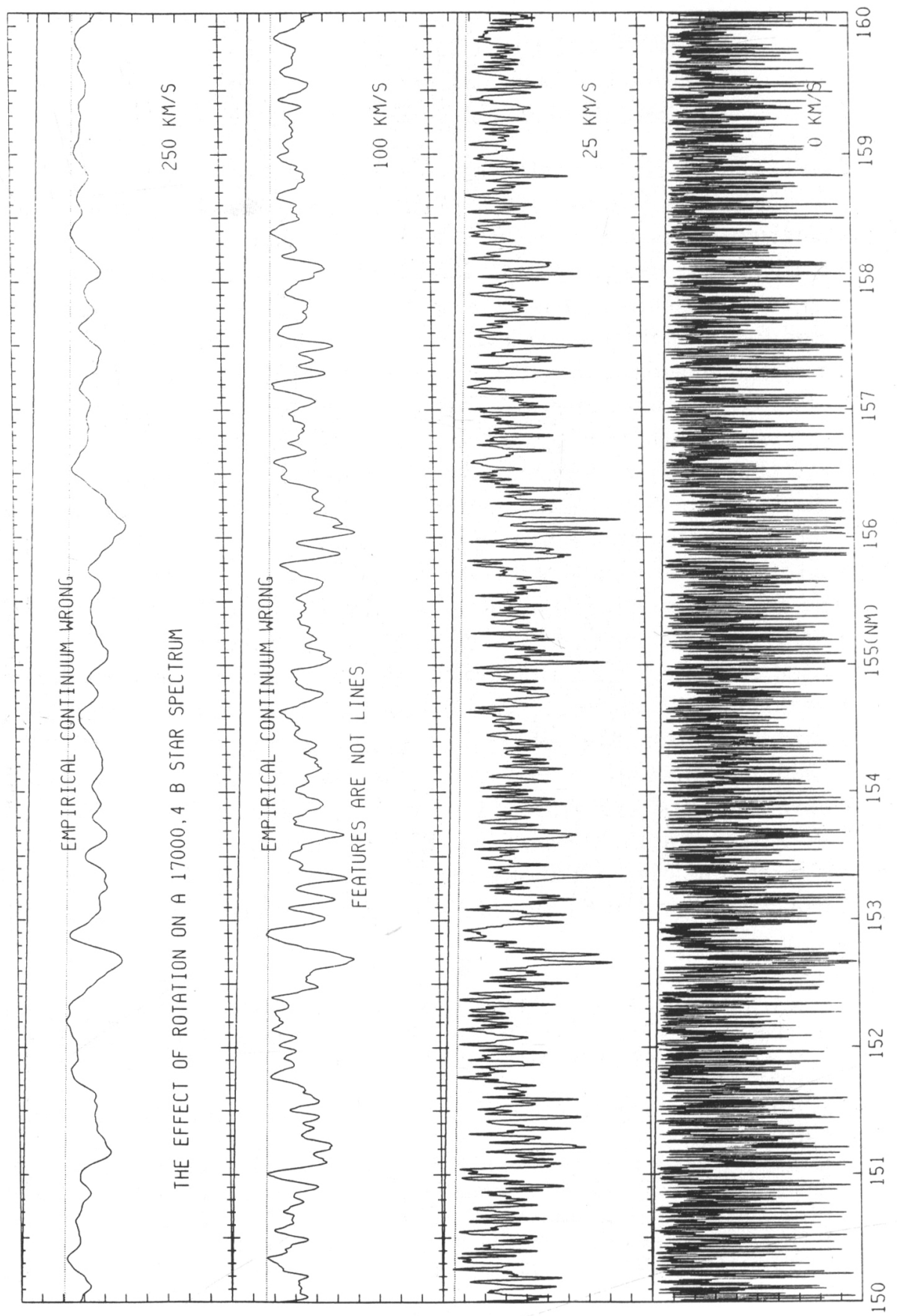

Figure 1. 


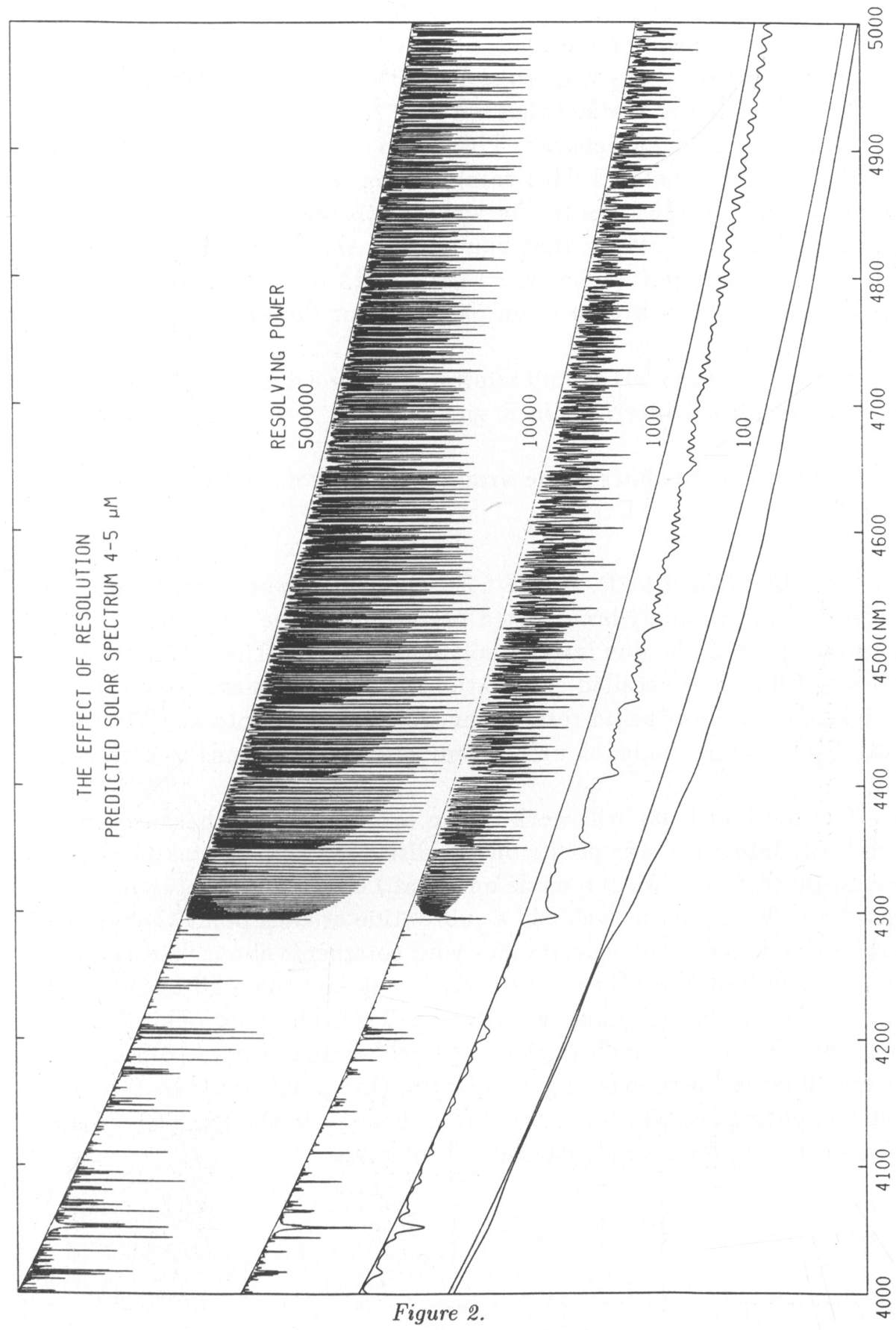


R.A. BELL to B. KURUCZ Q. I would like to thank you for your work and giving me your line data list.

However, I must say that your $4210^{* *} 6$ atomic line list contents many errors in addition to the fact that only $10^{* *} 6$ of them occur in the spectrum of the Sun, Vega or Aldebaran. The errors are shown in a paper (MNRAS 268,771), which contains data comparisons with the solar spectrum. You predicted about 115 lines in the wavelength region $4600-4650 \mathrm{~A}$, only 90 are observed. The reason that you predict the solar UV flux is that you have many too lines. These errors cast doubt on your models. The Buser and Kurucz colours are based in part on your fluxes and must be treated with caution.

Finally your models should allow for stellar evolution e.g. include $\mathrm{C}$ depletion and $\mathrm{N}$ enhancement in $\mathrm{K}$ giant stars.

A. If you have many lines in the wrong place you must be using the predicted line list.

M. DWORETSKY to R. KURUCZ Q. I have experimented with calculations of rotationnaly broadenned profiles using the "old" methods (using accurate limb-darkening laws in polynomial form). The difference between these profiles and detailed summations of intensities are very small up to $100 \mathrm{~km} / \mathrm{s}$. For more rapid rotators one needs to take into account the physical distorsion of shape, as well as temperature variations over the surface.

A. The method I use will work for an arbitrary shape because I just numerically integrate over points on the disk. I have been making the approximation that the disk is a circle and that I only have to integrate over one quadrant. I can just as well call a subroutine at each point that returns an arbitrary intensity and velocity. For your comments about "old-fashionned" broadening functions, they are clearly wrong and the high-to-noise (1000s) are resolution where I make tests.But I will consider your point that the old way may be good enough in the real world so that I need to produce only $0 \mathrm{~km} / \mathrm{s}$ flux and a broadening programm. One point needs to be clarified I am computing the whole spectrum from the UV to the IR. The old method can work only for a small wavelength intervals. 